\title{
Location-Aware Fast Link Switching Scheme for Visible Light Communication Networks
}

\author{
Tuan Nguyen*, Mostafa Zaman Chowdhury*, Yeong Min Jang
}

\begin{abstract}
Quality of Service (QoS) provisioning is an important issue in the design of next generation wireless network. In visible light communication (VLC) networks, link switching is a solution to maintain or improve the quality of communication. In this paper, we propose a novel link switching scheme using the location of mobile nodes (MNs). The current serving transmitter uses location history of the MN to find out which neighbor transmitter the $\mathrm{MN}$ is approaching. This neighbor transmitter is chosen to inform the $\mathrm{MN}$ as the next serving transmitter. The simulation results show that our proposed scheme gains better performance than non-predictive link switching scheme.
\end{abstract}

Key words : Link switching, VLC, location awareness, and delay.

\section{I . Introduction}

According to recent study [1], indoor coverage holes in cellular systems have a significant effect on customer's satisfaction. Thus, indoor communication systems have been researched and developed strongly to connect users with diverse capabilities and services. VLC has been emerging as a new promising technology that can supply a ubiquitous information communication service in indoor environment. VLC transmits data by intensity modulating optical sources, such as light-emitting diodes (LEDs) and laser diodes (LDs), which are faster than the persistence of the human eye. VLC merges lighting and data communications in applications such as area lightings, signboards, streetlights, vehicles, and traffic signals ${ }^{[2]}$. VLC provides many advantages such as visibility, high SNR (Signal to Noise Ratio), free license frequence band, high security, easy implementation, and freedom of interference from radio.

Nowadays, the interest in VLC using white LEDs is gradually growing as necessity for indoor communication systems because there are many devices using the lighting in our offices, homes, traffic signals, home appliances including TVs, and etc $^{[3]}$. A challenging issue which needs to be considered in VLC networks is the guarantee of seamless connectivity when a MN moves from the coverage area of a transmitter to the coverage area of another one, as known as link switching. The system needs fast link switching scheme to maintain the connectivity between the transmitter and the MN. In this paper, we propose in literature a new link switching scheme for VLC networks. In our scheme, the serving transmitter finds out the neighbor transmitters that the $\mathrm{MN}$ is approaching. The MN only scans these neighbor transmitters. Hence, the scanning time in link switching procedure is reduced, since then the

※ This work was supported by the IT R\&D program of MKE/KEIT [10035362, Development of Home Network Technology based on LED-ID].

※ This work was also supported by research program 2012 of Kookmin University in Korea.

- 주저자 : Department of Electronics Engineering, Kookmin University, 준회원

교신저자 : Department of Electronics Engineering, Kookmin University, yjang@kookmin.ac.kr, 종신회원

* Kookmin University

논문번호 : KICS2012-06-276, 접수일자 : 2012년 6월 10일, 최종논문접수일자 : 2012년 10월 11일 
link switching delay is reduced as well.

The rest of this paper is organized as follows. The non-predictive link switching scheme is given in Section II. In Section III, we present our proposed link switching scheme. In Section IV, we evaluate the performance of our proposed scheme. Finally, conclusions are discussed in Section V.

\section{Non-predictive Link Switching Scheme}

Link switching is the procedure whereby a $\mathrm{MN}$ changes its communication link within a coverage area of transmitter or from one transmitter to another transmitter via an alternative link. This procedure is necessary to maintain or improve the quality of communications due to interference or mobility of the MN. To understand the link switching procedure, we consider the scenario with two transmitters and one $\mathrm{MN}$ moving from coverage area of serving transmitter (for instance, Transmitter \#1) to target transmitter's (for instance, Transmitter \#2). When a MN moves to boundary area, the strength of the received signal is decreased. Also MN receives another signal from another transmitter in the boundary area. At that time, link is disconnected from serving transmitter and synchronization process is implemented for connecting to the target transmitter.

The link switching procedure is shown in Figure 1. At the initial of synchronization process, the $\mathrm{MN}$ broadcasts its information including MN's ID. If one transmitter receives broadcast information message, it sends detect device message to the MN whose ID has been included in the message. If the $\mathrm{MN}$ responds with $A C K$ message, the transmitter sends boundary information to the MN. The boundary information includes some parameters which are used for links switching decision of $\mathrm{MN}$.

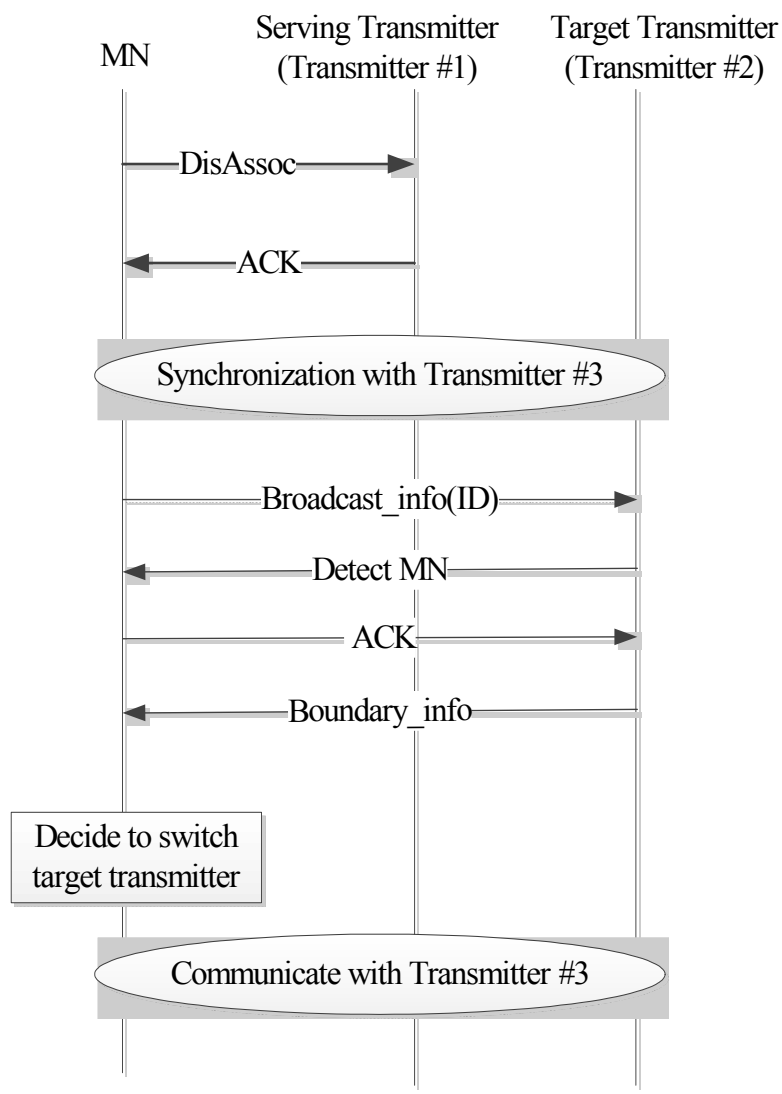

Fig. 1. Non-predictive link switching procedure.

After synchronization process is completed, the MN gets all transmitter information and makes decision to communicate with one of them. Making decision can depend on priority mechanism of MN. This mechanism helps the MN to choice the best case for data transfer.

After finishing the decision process, the $\mathrm{MN}$ tries to communicate with the chosen transmitter. Finally, when the reentry process is successful, the $\mathrm{MN}$ continues to receive data from new serving transmitter.

\section{Proposed Link Switching Scheme}

In this section, we describe the detail of location estimation using Time Difference of Arrival (TDOA) method as well as our proposed link switching scheme. Our scheme aims to reduce the scanning time of link switching procedure. 


\subsection{Location Estimation using TDOA method}

In order to use a location-aware scheme, the location of each user in the VLC environment needs to be determined. The location of a $\mathrm{MN}$ can be determined using various types of measurement, such as Global Positioning System (GPS) [4], Received Signal Strength Indication (RSSI) [5], Time Difference of Arrival (TDOA) [6], Angle of Arrival (AOA) [7], and etc. TDOA method can accurately obtain the estimated location of user by using the knowledge of coordinates of three different benchmarks or reference points. In this paper, we use TDOA measurement to estimate the location of MNs. We denote the two-dimension coordinate of a $\mathrm{MN}$ at time $t_{k}$ and the coordinates of the three reference transmitters as $\left(x_{k}, y_{k}\right),\left(x_{1}, y_{1}\right),\left(x_{2}, y_{2}\right)$, and $\left(x_{3}, y_{3}\right)$, respectively. So, the value of $\left(x_{k}, y_{k}\right)$ can be estimated by using these following equations:

$$
\left\{\begin{array}{l}
c\left(t_{2}-t_{1}\right)=\sqrt[2]{\left(x_{k}-x_{2}\right)^{2}+\left(y_{k}-y_{2}\right)^{2}}-\sqrt[2]{\left(x_{k}-x_{1}\right)^{2}-\left(y_{k}-y_{1}\right)^{2}} \\
c\left(t_{3}-t_{1}\right)=\sqrt[2]{\left(x_{k}-x_{3}\right)^{2}+\left(y_{k}-y_{3}\right)^{2}}-\sqrt[2]{\left(x_{k}-x_{1}\right)^{2}-\left(y_{k}-y_{1}\right)^{2}}
\end{array}\right.
$$

where $\mathrm{c}$ is the speed of light, $t_{i}$ is the transmission time from Transmitter \#i to the $\mathrm{MN}(\mathrm{i}=1,2,3)$.

The problem of above TDOA method is that when a $\mathrm{MN}$ cannot receive signal from one of three transmitters (due to obstacle between $\mathrm{MN}$ and transmitter, unability of transmitter's operation, and etc.), the position estimation algorithm using three transmitters becomes unavailable. To cope with this problem, we use previous locations of $\mathrm{MN}$ together with two remaining transmitters. Because the $\mathrm{MN}$ only can receive signal from two remaining transmitters (for instance, Transmitter \#2 and Transmitter \#3), the following equations can be obtained:

$$
\left\{\begin{array}{l}
\sqrt[2]{\left(x_{k}-x_{2}\right)^{2}+\left(y_{k}-y_{2}\right)^{2}}=c\left(t_{t 2}-t_{0}\right) \\
\sqrt[2]{\left(x_{k}-x_{3}\right)^{2}+\left(y_{k}-y_{3}\right)^{2}}=c\left(t_{t 3}-t_{0}\right)
\end{array}\right.
$$

where $t_{0}$ is the time at which a transmission took place at the $\mathrm{MN}$ and $t_{t i}$ is the time at which
Transmitter $\# i$ received signal from $\mathrm{MN}(i=2,3)$. From Equation (2) we can achieve two roots. One of these two roots is exact estimated location of the $\mathrm{MN}$ in case that the $\mathrm{MN}$ can receive 3 signals from 3 transmitters. The exact root can be found out by checking following criteria:

- In a small interval, the variation of MN's coordinate from time $t_{k-1}$ to time $t_{k}$ is similar to its variation from time $t_{k-2}$ to time $t_{k-1}$.

- In a small interval, the variation of MN's velocity is very small. Since then the variation of distance between two consecutive locations is also very small. Thus, the exact root is the one whose distance to MN's previous location at time $t_{k-1}$ is more similar to the distance from MN's previous location at time $t_{k-2}$ to its previous location at time $t_{k-1}$.

After a certain interval, the $\mathrm{MN}$ stores its current location into a location database. This database is used by MN's current serving transmitter to predict the next serving transmitter for $\mathrm{MN}$.

\subsection{Proposed Link Switching Scheme}

In the non-predictive link switching scheme, a MN has to scan all the neighbor transmitters to find out the best one as the next serving transmitter. This process takes a long time to finish. Our proposed scheme aims to reduce scanning time, so the link switching delay is decreased.

When a MN moves to the boundary area of the serving transmitter, the strength of the received signal is decreased. Also the MN receives other signals from other neighbor transmitters in the boundary area. If the received signal from the serving transmitter becomes too weak (below a link switching threshold), the link switching procedure starts. First, the MN sends Link_SW_REQ message, which contains its location information as mentioned before, to the serving 


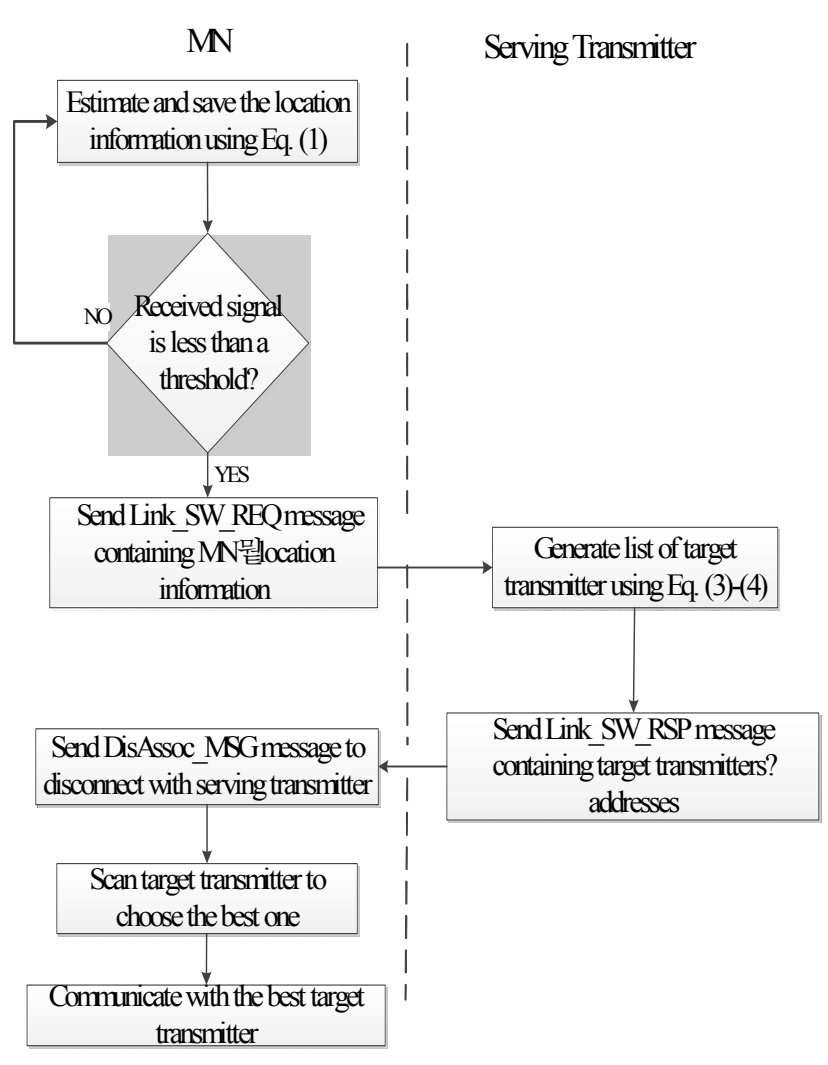

Fig. 2. Location-aware link switching procedure.

transmitter. Based on location information from the MN, the serving transmitter determines the neighbor transmitters to which the $\mathrm{MN}$ is approaching. To achieve this one, we denote the distance from the $\mathrm{MN}$ to Transmitter $\# \mathrm{j}$ at time $t_{k-1}$ and $t_{k}$ as $d_{k-1, j}$ and $d_{k, j}$, respectively. So, the variation of distance from the $\mathrm{MN}$ to Transmitter $\# \mathrm{j}$ at time $t_{k}$ can be calculated by:

$$
\Delta_{k+1, j}=d_{k, j}-d_{k-1, j}
$$

If $\Delta_{k+1, j}<0$, then we say that the $\mathrm{MN}$ is approaching Transmitter \#j. In contrast, if $\Delta_{k+1, j}>0$, we say that the MN is moving far away from Transmitter \#j. We denote the total number of neighbor transmitters to which the $\mathrm{MN}$ is approaching as $N_{\text {candidate. }}$ The serving transmitter chooses a number of candidate transmitters which have the closer distances to the MN's location at time $t_{k}$ as the target transmitters. This set, denoted as $N_{\text {target }}$, is given by:

$$
N_{\text {target }}=\left\lceil n \times N_{\text {candidate }}\right\rceil
$$

where $0<n \leq 1$. After that, the serving transmitter sends Link_SW_RSP message that contains addresses of target transmitters to the MN. Based on this information, the MN sends back DisAssoc_MSG message to disconnect with serving transmitter and start scanning the target transmitters. After that, based on the received signal strength, the $\mathrm{MN}$ chooses the best target transmitter as the next serving transmitter. Finally, the MN starts communicating with the best target transmitter. Figure 2 shows the flowchart of our proposed link switching scheme.

\section{Performance Analysis}

To evaluate the proposed link switching scheme, we choose OMNET, one of believable network analyzing tools. OMNET is open source software, so it is possible to modify the network due to specific purpose. We evaluate our proposed scheme with the different cases of value of $n$ in Equation (4). This value affects the number of target transmitters chosen for $\mathrm{MN}$ to scan, since then the performance of the proposed scheme is affected. We consider three cases of the value of $n$ for the performance evaluation: $n=1$ (all the candidate transmitters are chosen), $n=1 / 2 \quad$ (a half of the number of candidate transmitters are chosen), and $n=1 / N_{\text {candidate }}$ (only the closest candidate transmitter is chosen).

Figure 3 shows the relation between link switching delay and the number of candidate transmitters in the boundary area. The link switching delay, denoted as $T_{L S W}$, can be calculated as:

$$
\begin{aligned}
T_{\text {LSW }}= & T_{\text {req }}+T_{\text {disassoc }}+T_{\text {scanning }}+ \\
& T_{\text {authen }}+T_{\text {slot_alloc }}+T_{\text {assoc }}
\end{aligned}
$$

where:

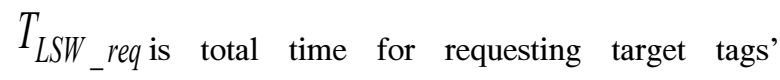
addresses, 


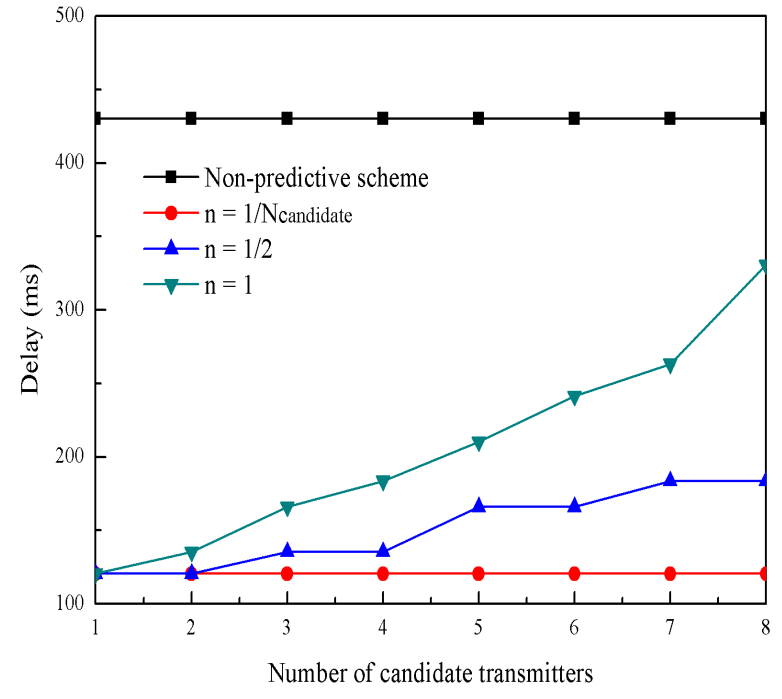

Fig. 3. Link switching delay vs. number of candidate transmitters in the boundary area.

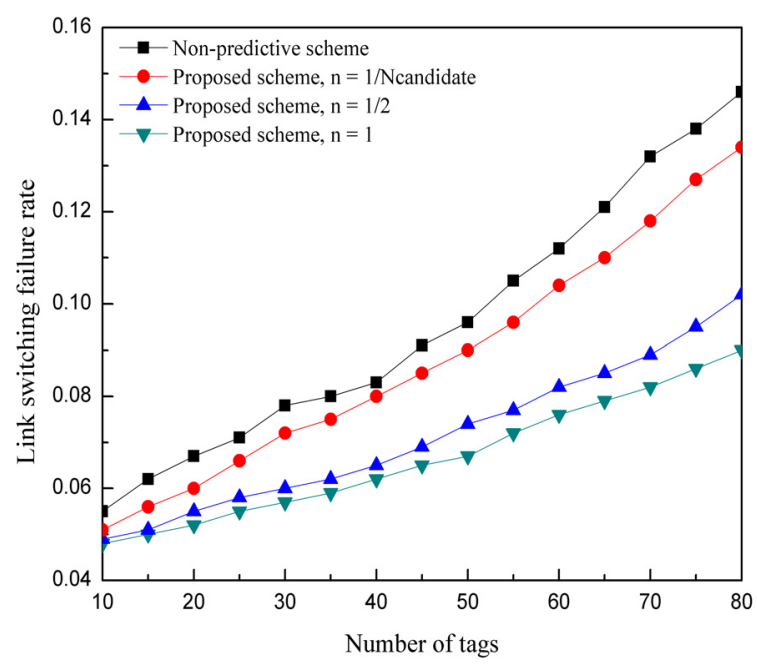

Fig. 4. Link switching failure rate vs. number of transmitters in the boundary area.

$T_{L S W \_d i s a s s o c}$ is total time for disassociation with current serving tag,

$T_{\text {scanning is total time for scanning target tags, }}$

$T_{\text {authen is total time for authentication with selected }}$ target tag,

$T_{\text {slot_alloc is total time for slot-allocation with next }}$ serving tag,

$T_{\text {assoc }}$ is total time for association with next serving tag.

Due to the nature of non-predictive link switching scheme that scans all the transmitters to find the best one, the link switching delay of this scheme is constant in any case of candidate transmitters' number (430.15 ms). By decreasing the number of scanned transmitters in the scanning period, our proposed scheme efficiently reduces the switching delay. In case of $\mathrm{n}=1 / N_{\text {candidate }}$ (which means the $\mathrm{MN}$ only scans the closest candidate transmitter), the link switching delay is $120.5 \mathrm{~ms}$ and independent of the number of target transmitters.

Figure 4 compares the link switching failure rate of the non-predictive scheme with our proposed scheme. A link switching process is considered as failed when the whole procedure does not finish within a predefined threshold level. The success of a link switching process can be expressed as follows:

$$
T_{\text {process }} \leq \gamma
$$

where $T_{\text {process }}$ is link switching processing time and $\gamma$ is failure rate time threshold. In this paper, $\gamma$ is set to be $0.7 \mathrm{~s}$.

When the number of transmitters covering same areas is increased, MNs have to scan more transmitters in link switching procedure. Thus, link switching processing time is increased, since then the link switching failure rate is increased as well.

Besides, the value of $n$ in Equation (4) affects link switching failure rate. When $n$ is increased, the number of neighbor tags that the serving tag chooses to inform the MN is increased. Thus, the number of scanned transmitters in the scanning period is increased, since then link switching processing time, as well as link switching failure rate, is increased as well.

\section{Conclusions}

Using location information of mobile devices is an effective way to improve the performance of communication systems. In this paper, we propose a new link switching scheme for VLC networks. The locations of MNs are used to assist MNs in choosing the new serving transmitter when they need to proceed link switching procedure. Our proposed scheme outperforms the conventional scheme in reducing link switching delay and link switching failure rate. 


\section{References}

[1] Do. N. Knisely, T. Yoshizawa, and Frank Favichia, "Standardization of femtocells in 3GPP”, IEEE Communications Magazine, vol. 47, no. 9, pp. 68-75, Sept., 2009.

[2] IEEE 802.15.7, "IEEE standard for local and metropolitan area networks - Part 15.7: Short-Range Wireless Optical Commnucation Using Visible Light", 2011.

[3] T. Komine and M. Nakagawa, "Fundamental analysis for visible-light communication system using LED lights", IEEE Transactions on Consumer Electronics, vol. 50, no. 1, pp. 100-107, Feb., 2004.

[4] N. M. Drawil and O. Basir, "Intervehicle-communication-assisted localization", IEEE Transactions on Intelligent Transportation Systems, vol. 11, no. 3, pp. 678-691, Sept., 2010.

[5] S. Mazuelas et al., "Robust indoor positioning provided by real-time RSSI values in unmodified WLAN networks", IEEE Journal of Selected Topics in Signal Processing, vol. 3, no. 5, pp. 821-831, Oct., 2009.

[6] S. Jung, S. Hann, and C. Park, "TDOA-based optical wireless indoor localization using LED ceiling lamps", IEEE Transactions on Consumer Electronics, vol. 57, no. 4, pp. 1592-1597, Nov., 2011.

[7] M. Alsehaili, S. Noghanian, D. A. Buchanan, and A. R. Sebak, "Angle-of-arrival statistics of a three-dimensional geometrical scattering channel model for indoor and outdoor propagation environments", IEEE Antennas and Wireless Propagation Letters, vol. 9, pp. 946-949, 2010.

\section{Tuan Nguyen}

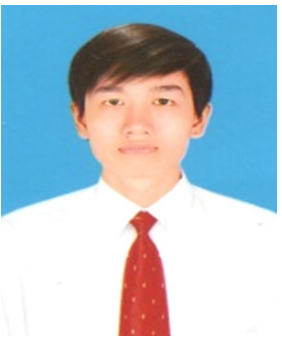

He received his B.Sc. in Electronics and Telecommunications from Hanoi University of Science and Technology (HUST), Vietnam in 2011. Currently he is working towards his M.Sc. degree in the department of Electronics Engineering at the Kookmin University. His research interests include convergence networks, VLC networks, LED-ID networks, and QoS provisioning.

\section{Mostafa Zaman Chowdhury}

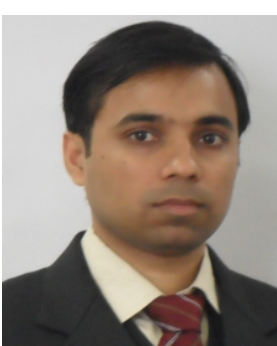

$\mathrm{He}$ received his B.Sc. in Electrical and Electronic Engineering (EEE) from Khulna University of Engineering and Technology (KUET), Bangladesh in 2002. He received his M.Sc. and $\mathrm{PhD}$ in Electronics Engineering from Kookmin University, Korea in 2008 and 2012, respectively. His research interests include convergence networks, VLC networks, QoS provisioning, mobile IPTV, and femtocell.

\section{Yeong Min Jang}

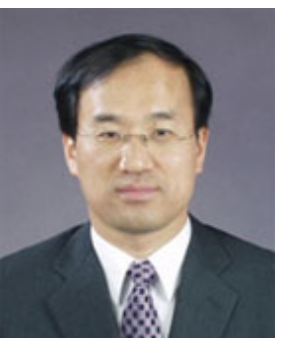

He received the B.E. and M.E. degree in Electronics Engineering from Kyungpook National University, Korea, in 1985 and 1987, respectively. $\mathrm{He}$ received the doctoral degree in Computer Science from the University of Massachusetts, USA, in 1999. He worked for ETRI between 1987 and 2000. Since Sept. 2002, he is with the School of Electrical Engineering, Kookmin University, Korea. His research interests are $5 \mathrm{G} / \mathrm{B} 4 \mathrm{G}$, radio resource management, LED-ID, VLC networks, multi-screen service, and small cell networks. 